\title{
Millimetre Wave Emission by Discrete Method in Sea- foam Layer at WindSat Frequencies
}

\author{
Ayibapreye Kelvin Benjamin ${ }^{*}$, Aguiyi Nduka Watson ${ }^{* *}$, Godday Biowei ${ }^{* * *}$ \\ * Department of Electrical/Electronic Engineering, Niger Delta University \\ ** Department of Electrical/Electronic Engineering, Federal University Otueke \\ *** Department of Electrical/Electronic Engineering Niger Delta University
}

DOI: 10.29322/IJSRP.12.01.2022.p12146

http://dx.doi.org/10.29322/IJSRP.12.01.2022.p12146

\begin{abstract}
Computation of millimetre wave scattering by sea foam is a significant query which contributes immensely to measurement of sea surface emissivity and brightness temperature by passive microwave radiometers. For efficient evaluation of scattering by foam covered sea surface and measurement of brightness temperatures in milli-Kelvins, a discrete based physical model of sea foam is developed to provide accurate estimate of the complex effective dielectric constant of sea foam. The sea foam layer is modelled as sequences of thin phase screens in two dimensional $(2 \mathrm{D})$ slices of sea foam layer with equal depth $d(\mathrm{~mm})$. Each layer comprised of random distribution of bubbles that follows a log-normal distribution pattern with geometrical and optical properties such as foam layer thickness, foam void fraction, foam volume fraction, sea surface temperature and sea surface salinity. Results of sea surface emissivity as a function of polarization, angle of incidence, WindSat frequencies and thickness of sea foam are presented.
\end{abstract}

Index Terms- Discrete method, millimetre wave emission, sea foam, WindSat frequencies

\section{INTRODUCTION}

$\mathrm{B}$ ubble distribution and its Climatologies in the open ocean are dependent on acoustical and open measurements of bubbles. The dominance of wave breaking to windspeed determines global bubble distribution. Bubbles plays a vital role in the air-sea exchange of heat and particles as important component of geochemical cycling via their transport of material in the open and surface micro-layer. The bubble size distribution is a major parameter for effective evaluation of millimetre wave propagation in randomly distributed media. This presents a research problem of developing a physical based model of sea surface covered by foam or distribution of bubbles that is most suitable for evaluation of millimetre wave scattering and emissivity of sea foam. Wilheit, Pandey and Kakar mentioned several empirical models for evaluation of sea foam emissivity and brightness temperature, which was based on experimental data of sea surface by foam, as function of angle of incidence and frequency [1-4]. William proposed that foam emissivity is strongly dependent on foam thickness at X-band in a waveguide [5]. It was demonstrated that foam microstructures and air volume fraction (AVF) are significant in evaluating sea foam emissivity [6], [7]. These factors were further validated by controlled experiments to measure seawater coating thickness, bubble size distribution, and foam layer thickness [8], [9]. The aforementioned empirical models could not explain theoretically, how a foam layer influence microwave radiation. This all-important puzzle necessitates the development of theoretical models which unveil the effects of foam layer to sea surface emission. Theoretical models such as the vector radiative transfer (VTR) equation [10], [11] the effective medium approximation (EMA) theory, and the dense media transfer (DMRT) method were used to investigate emissivity and scattering by sea foam covered sea surface $[12,13,14,15,16,17,18]$. Reasonable results were obtained using these theoretical models but the interactions of air-bubbles coated with thin layer of seawater are not considered precisely in the sea foam complex dielectric constant within millimetre wave by a suitable theory. The effective dielectric constant of a dense media remains a significant parameter in estimating microwave radiation by sea foam. Hence, there is need to develop a more accurate model for estimating the effective dielectric constant of sea foam layer. 
In recent times, mixing rules or effective medium theories provide a systematic insight for calculating the effective dielectric constant (permittivity) of sea foam (whitecaps) at WindSat frequencies $(10.7 \mathrm{GHz}-37 \mathrm{GHz}$ ) [19]. Electromagnetic wave scattering by foams are weak at these frequencies which lead to the use of as Maxwell Garnett, Bruggeman, Coherent potential, Looyenga, and Refractive models in evaluating the effective dielectric constants of sea-foams [19].

This was motivated by past reports on various heterogeneous dielectric mixtures and properties of sea-foam which are important in order to calculate the effective dielectric constants of sea-foams. There are few measurements characterizing the sea-surface covered by foam layers and commonly simulated artificial sea-foam but numerous experimental and field observations which characterized deep plumes well [6, 8, 9, 20,21]. Recent model in [22] eliminated a major contributor to absorption (the water below the foam) and considered large thick- walled bubbles at relatively high frequency. This unsurprisingly, predicts low absorption (about $28 \%$ of the total extinction) and significant scattering, which appear consistent with the other analytical and experimental findings.

The Effective impedance model (EIM) for estimation of the effective permittivity of diphasic dielectrics is based on an equivalent impedance circuit as a function of frequency with complex shaped inclusions. Here, the dielectric composite is discretized into partial impedances, specifically, R-C elements which is equivalent to lossy capacitors. This model focuses on dielectric-dielectric mixtures, and works only in quasi-static conditions. The total impedance of the appropriate equivalent circuit is used to calculate the effective permittivity of the mixtures. This approach was applied to high permittivity inclusion in a low permittivity host in [23]. The geometry of the capacitor containing a composite dielectric is discretized into partial parallel-plate capacitor elements and the effective permittivity of the composite is obtained from the equivalent capacitance or impedance. The impedance or capacitance of an individual cell is described as a function of frequency and inclusion radius or volume fraction [24]. Validation of this method was implemented by [24] for prediction of effective permittivity of diphasic dielectrics using an equivalent capacitance method.

\section{DISCRETE METHOD OF COMPUTING SEA-FOAM EFFECTIVE DIELECTRIC CONSTANT}

The discrete method is implemented by considering 3-dimensional (3-D) sphere packing into a 3-D finite domain such as a cube/box. The bubble locations or positions are generated by uniformly distributed random numbers of size $N=1000000$ in $\mathrm{X}, \mathrm{Y}$, and $\mathrm{X}$ coordinates. Bubble radii $r_{i}$ of the $n$ randomly distributed air-bubbles are computed using the inverse method. The inverse method transforms the uniform variate $U(0,1)$ to normal variate with mean $\mu=0$ and variance $\sigma^{2}=1, N(0,1)$. Bubble radii $r_{i}$ and bubble size distribution $N(r)$ were computed assuming a mean $\mu=2.0$ and standard deviation $\sigma=0.5$, geometric mean $\mu_{g}=500 \mu m$ and geometric standard deviation $\sigma_{g}=2.0$, using an empirical expression for computation of the scaled normal variate and bubble size distribution (BSD) which is log-normally distributed. The 3-D randomly packed spheres in a cubic domain were translated into 2-D slices of solid annuli. The conversion from 3-D to 2-D slices was achieved by calculating the radius of each individual circle based on the concept of intersection of a sphere and a plane. The 2-D slices were discretized with grid sizes $\Delta x$ and $\Delta y$ which leads to intersection of circles bounded in a unit square with some grid points. The grid sizes were sampled such that the edges of the circle circumference which intersects with grid points farther from the inner grids bounded by the circles are negligible. We were able to estimate the effective dielectric constant by calculating the area of the outer and inner circles. The outer circle is seawater while the inner circle contains $80-$ $90 \%$ air, with known effective dielectric constants of air $\varepsilon_{\text {air }}=1.00005+0.0000 i$ and seawater at WindSat frequencies, we were able to estimate effective dielectric constant of $N$ randomly distributed spheres for 5 slices.

Table 1. Results for Dielectric constant of sea foam at frequencies of $10.7 \mathrm{GHz}$ and $37 \mathrm{GHz}$ for 52 -D Slices of randomly packed air-bubbles covered with thin-layer of seawater.

\begin{tabular}{|c|c|c|}
\hline FREQUENCY & $10.7 \mathrm{GHz}$ & $37 \mathrm{GHz}$ \\
\hline Slice 1 & $1.0948-0.1251 \mathrm{i}$ & $1.0006-0.0332 \mathrm{i}$ \\
\hline Slice 2 & $1.1248-0.1507 \mathrm{i}$ & $1.0108-0.0239 \mathrm{i}$ \\
\hline Slice 3 & $1.1622-0.1810 \mathrm{i}$ & $1.0225-0.0344 \mathrm{i}$ \\
\hline Slice 4 & $1.1983-0.2072 \mathrm{i}$ & $1.0315-0.0569 \mathrm{i}$ \\
\hline Slice 5 & $1.2271-0.2277 \mathrm{i}$ & $1.0465-0.0637 \mathrm{i}$ \\
\hline
\end{tabular}

This paper models the propagation phenomena that occurs when mmW at frequencies between $10.7 \mathrm{GHz}$ and $37 \mathrm{GHz}$ travels through a sea-foam layer $0 \geq Z \geq-d$, where $d$ is the depth of the sea-foam layer. The sea-foam layer is a diphasic composite with numerous isotropic-coated spherical particles, randomly embedded in an isotropic host with permittivity $\varepsilon_{f o a m}$ and no overlap between adjacent spherical air-bubbles. These air-bubbles consist of core air with permittivity $\varepsilon_{\text {air }}$ and coating shell with

This publication is licensed under Creative Commons Attribution CC BY.

http://dx.doi.org/10.29322/IJSRP.12.01.2022.p12146

WwW.ijsrp.org 
permittivity $\varepsilon_{\text {seawater }}$. For $N$ spherical particles with outer radii $r_{o N}$ and inner radii $r_{i N}$ randomly distributed in the host medium. The radii are represented by the bubble size distribution of the particles $N(r)$.

The simulation was also implemented for $N=1000000$. The log-normal distribution expressed as

$$
N(r)=\frac{1}{\ln \sigma \cdot r \sqrt{2 \pi}} \times \exp \left\{-0.5\left[\frac{\ln \frac{r}{\bar{r}}}{\ln \sigma_{g}}\right]^{2}\right\}
$$

$N(r)$ was calculated in Fortran adopting a geometric mean deviation $\sigma_{g}=2.0$ and mean radius $\bar{r}=500 \mu m$.

Equation (1) was compared with a scaled normal distribution with mean $=2.0$ and standard deviation $=0.5$. $\mathrm{X}=\sigma Z+\mu$, where $\mathrm{X}$ is the scaled normal variate, $\sigma$ the standard deviation and $\mu$ the mean.

Substitute $\mathrm{X}=\left\{\left[\frac{\ln \frac{r}{\bar{r}}}{\ln \sigma_{g}}\right]^{2}\right\}$ and was able to evaluate the Bubble radii $r$ as

$$
r=\bar{r} \cdot \exp \left(X \times \ln \sigma_{g}\right)
$$

Figure (2) shows the plot of bubble size distribution against bubble radii.

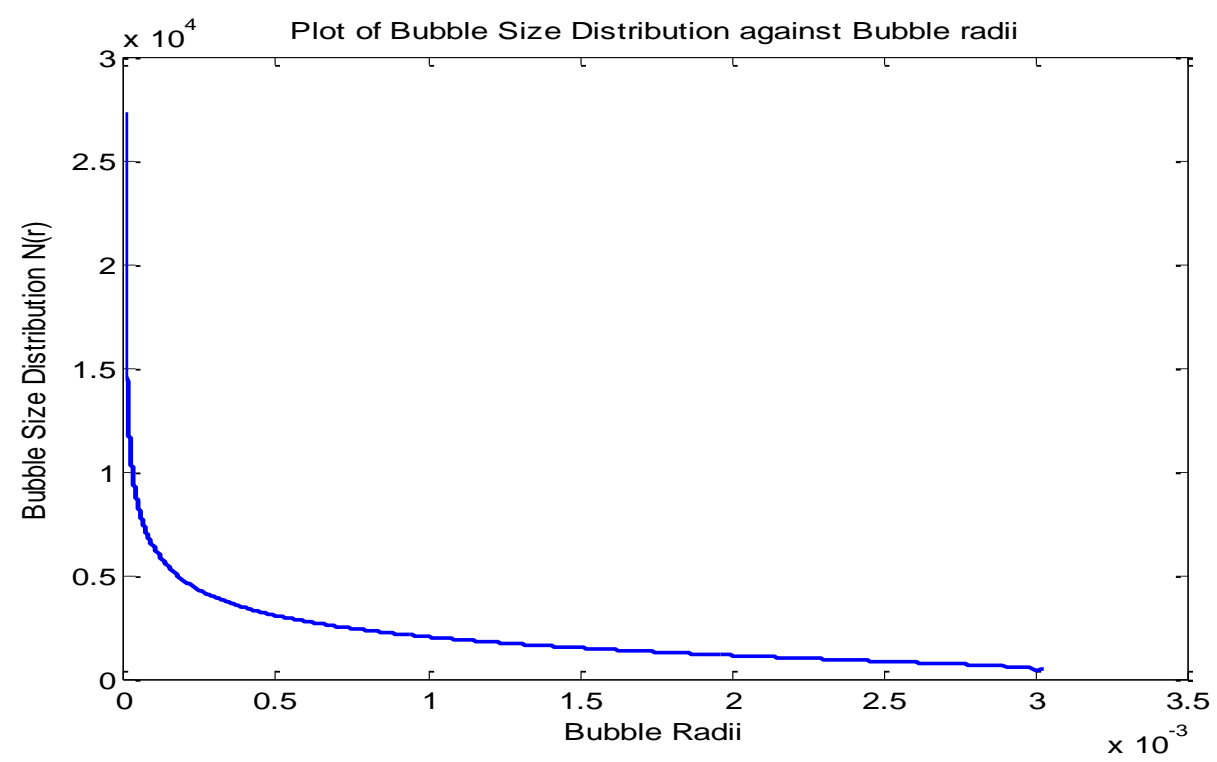

III. RESULTS AND FINDINGS

For fixed salinity $(34 \mathrm{psu})$, sea surface temperature $\left(20^{\circ} \mathrm{C}\right)$ and frequency range between $1.4 \mathrm{GHz}$ to $37.0 \mathrm{GHz}$, the dielectric constant of sea water was calculated by adopting existing methods by Stogryn, Guillou, Wentz, English, Klein and Swift [29]. These calculated dielectric constants were used to estimate the effective permittivity of sea foam. The area of the circles in each slice was calculated using the total number of grid points.

The effective dielectric constants of sea foams at frequencies $10.7 \mathrm{GHz}$ and $37.0 \mathrm{GHz}$ were calculated for 5 slices of randomly packed air-bubbles coated with thin layer of seawater. Table 1 shows variation of effective dielectric constant at 10.7 GHz and $37 \mathrm{GHz}$ for 5 2-D Slices of randomly packed air-bubbles covered with thin-layer of seawater. The effective dielectric constant of sea foam increases with increase in thickness of foam layer and decreases with increase in frequency as illustrated in Figure 5. The effective dielectric constant of sea foam is used in the computation of Fresnel's reflection coefficients for both horizontal $R_{p}^{\text {foam }}$ and vertical $R_{v}^{\text {foam }}$ polarized fields, at the air-foam interface and the foam-ocean interface. The Fresnel's reflection coefficients are used for the computation of sea surface emissivity $e^{\text {foam }}$ and brightness temperature $T_{B}$ in the radiative transfer equations given in [30]. 


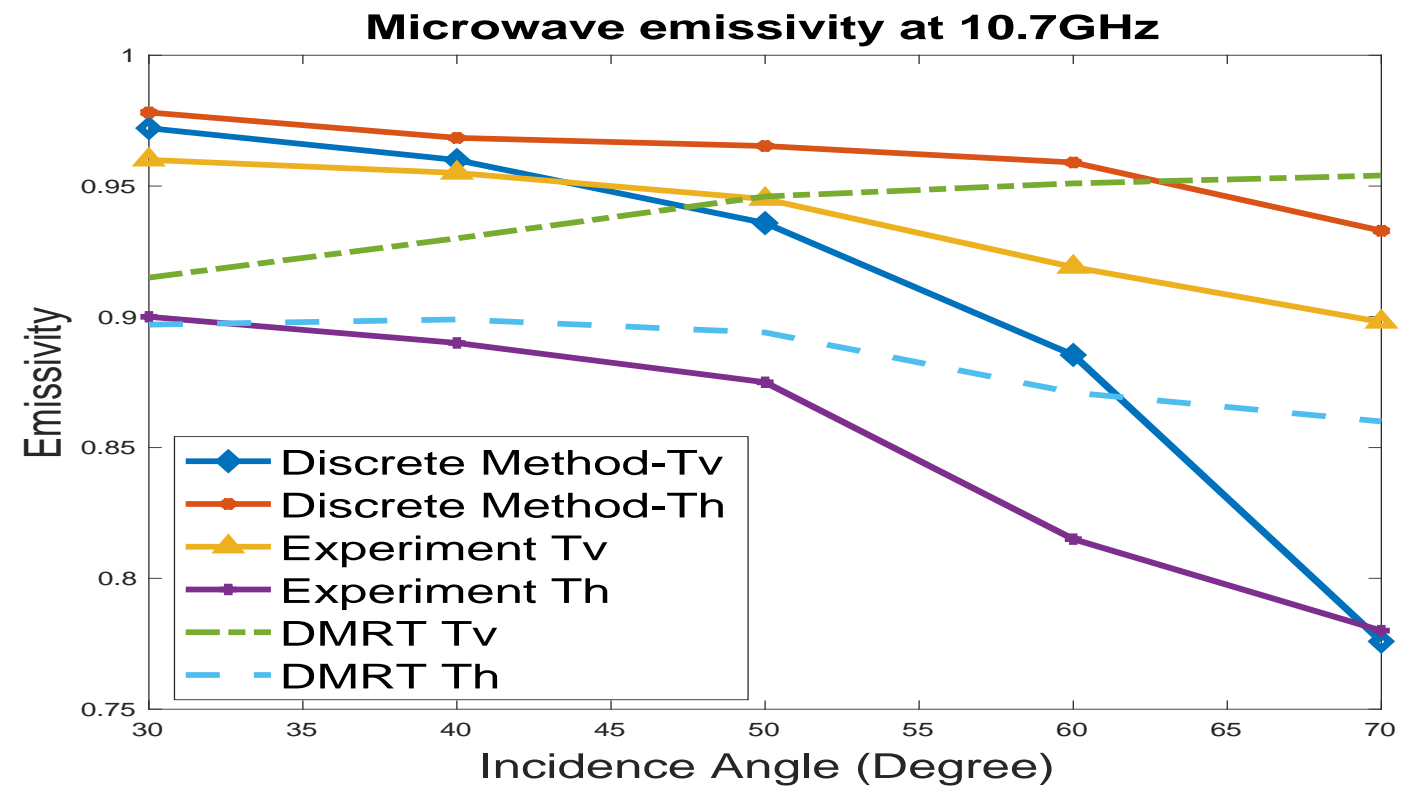

Figure 3. Comparison between simulation results and measurements of microwave emissivity at $10.7 \mathrm{GHz}$ for horizontal and vertical polarization as a function of incidence angle.

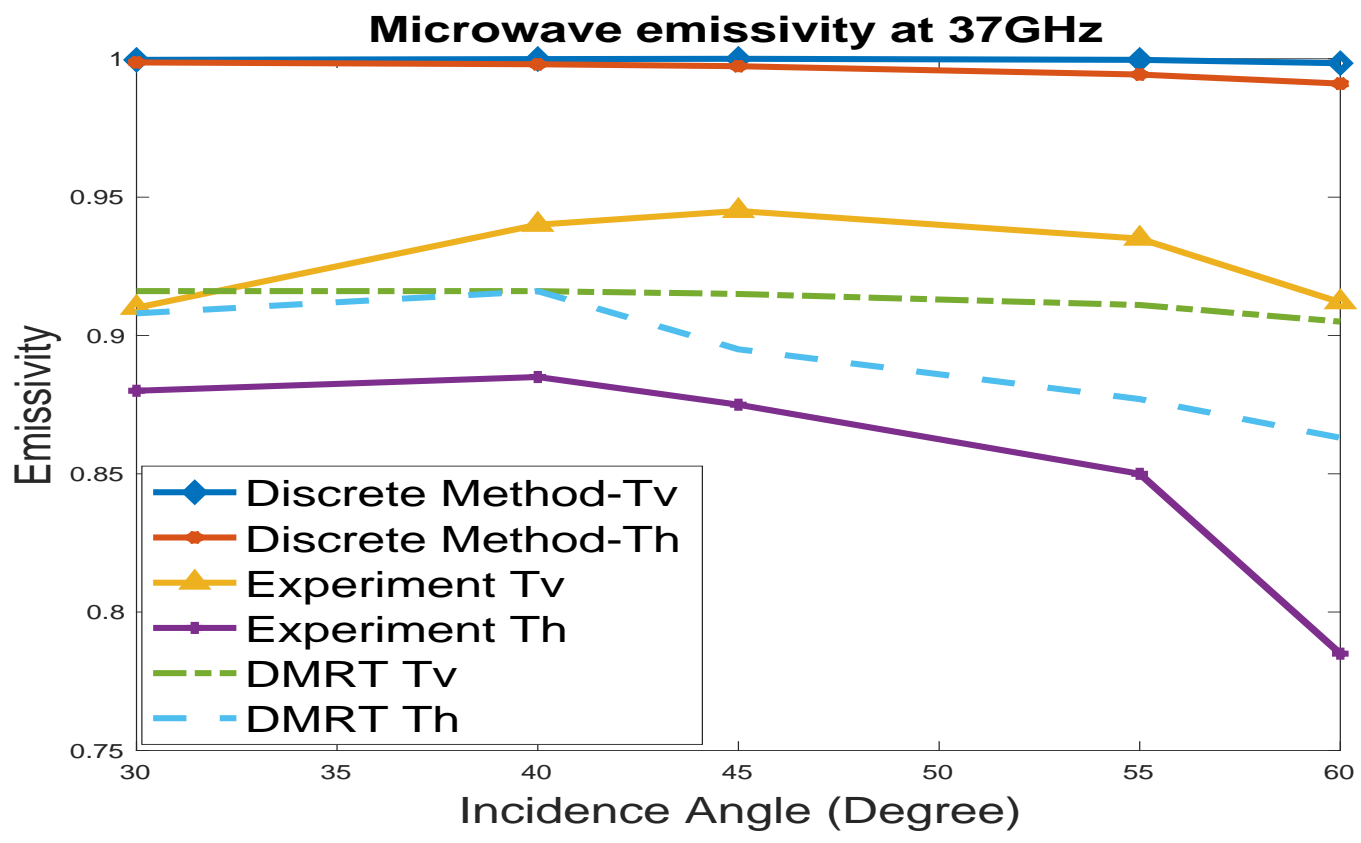

Figure 4. Comparison between simulation results and measurements of microwave emissivity at $37 \mathrm{GHz}$ for horizontal and vertical polarization as a function of incidence angle. 
Figures 3 and 4 show plots of microwave emissivity dependence on the incidence angles at $10.7 \mathrm{GHz}$ and $37 \mathrm{GHz}$ respectively, for both vertical and horizontal polarizations. These show that foam emissivity using experimental data, increases at angles $\theta_{i}=30^{\circ}, 35^{\circ}$ and $40^{\circ}$, then undergoes a decrease at angles $45^{\circ}, 50^{\circ}, 55^{\circ}$ and $60^{\circ}$ for both horizontal and vertical polarizations. The discrete method correspondingly, decreases with increase in angle of incidence for both horizontal and vertical polarizations at $10.7 \mathrm{GHz}$ and $37 \mathrm{GHz}$. The (DMRT) dense media radiative transfer yield results in reasonably good agreement with experimental measurements. The results show comparable emissivities at $10.7 \mathrm{GHz}$ and $37 \mathrm{GHz}$. Absorption effect at $37 \mathrm{GHz}$ is larger than $10.7 \mathrm{GHz}$ while scattering effect is more significant at $37 \mathrm{GHz}$. The results are in good agreement at small incidence angles. The disparity in results is explicit at larger angles of incidence.

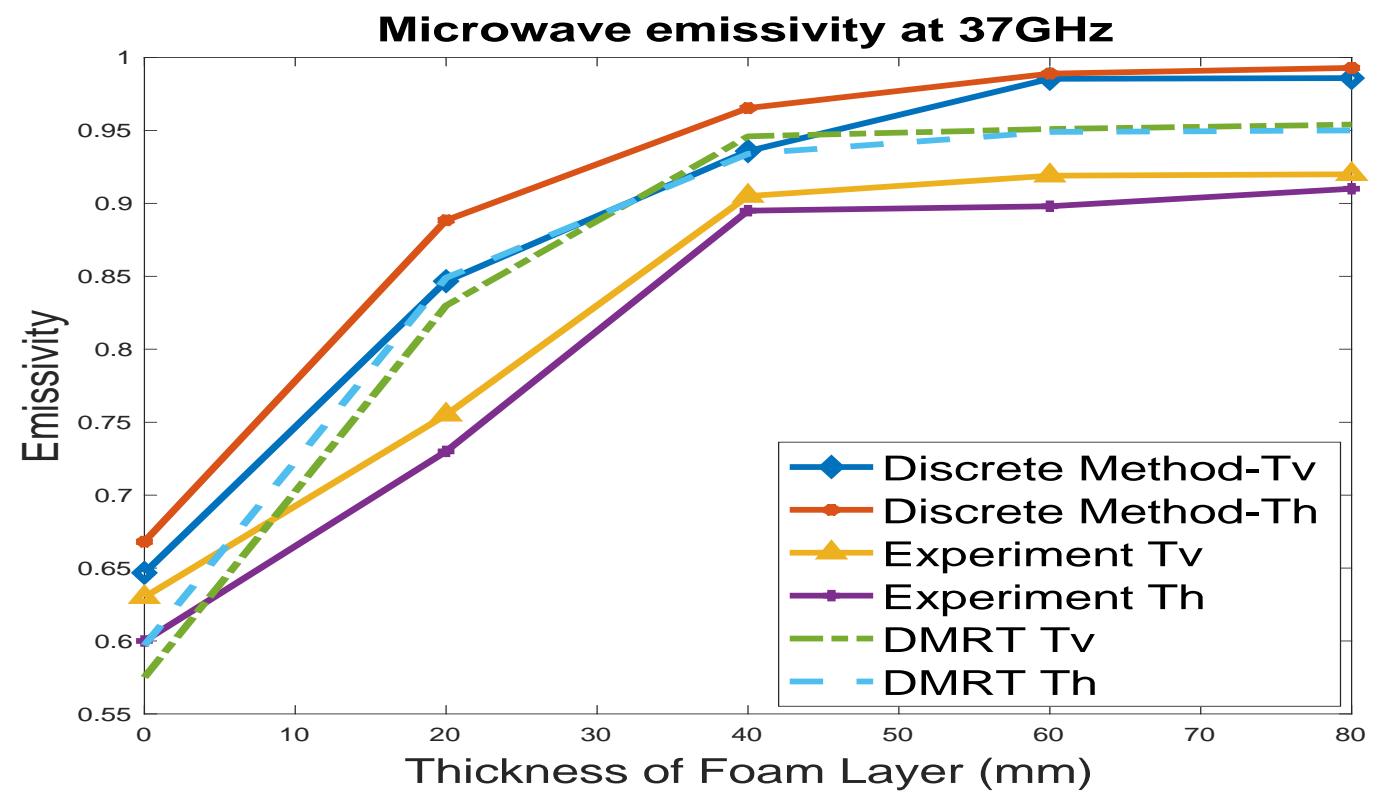

Figure 5. Comparison between simulation results and measurements of microwave emissivity at $37 \mathrm{GHz}$ for horizontal and vertical polarization as a function of foam layer thickness.

Figures 5 and 6 show the emissivity for horizontal and vertical polarization at WindSat frequencies $10.7 \mathrm{GHz}$ and $37 \mathrm{GHz}$, with dependence on foam layer thickness for randomly distributed air bubbles at incidence angle $\theta=53^{\circ}$. The coated bubbles are $80-95 \%$ of actual foam radii. We used foam parameters; mean bubble radius $r_{\text {mean }}=500 \mu \mathrm{m}$, minimum bubble radius $r_{\text {min }}=6.9 \mathrm{~mm}$ and maximum bubble radius $r_{\max }=5.5 \mathrm{~cm}$. Scattering increases with particle size and the effective scattering mean size of the DMRT and experimental models was reported substantially larger than the mean size of the radius. The discrete method used randomly distributed size particles with mean radius $r_{\text {mean }}=500 \mu \mathrm{m}$, which was chosen as it's comparable to bubble radius used by Chen et.al, which represent the effective mean scattering. In actual foam, the coating thicknesses vary as a function of foam depth. Figures 5 and 6 show that scattering effect increases with increase in size of bubbles, and the albedo increases also which leads to decrease in brightness temperatures. The figure 5 and 6 shows explicitly that emissivity increases as the foam layer thickness increases and later saturates at a specific foam thickness, for both horizontal and vertical polarization. Chen et.al DMRT simulation results show that the saturation point of horizontal polarization was slightly larger than that of vertical polarization. The discrete method results show reasonably good agreement with DMRT and experimental measurements. 


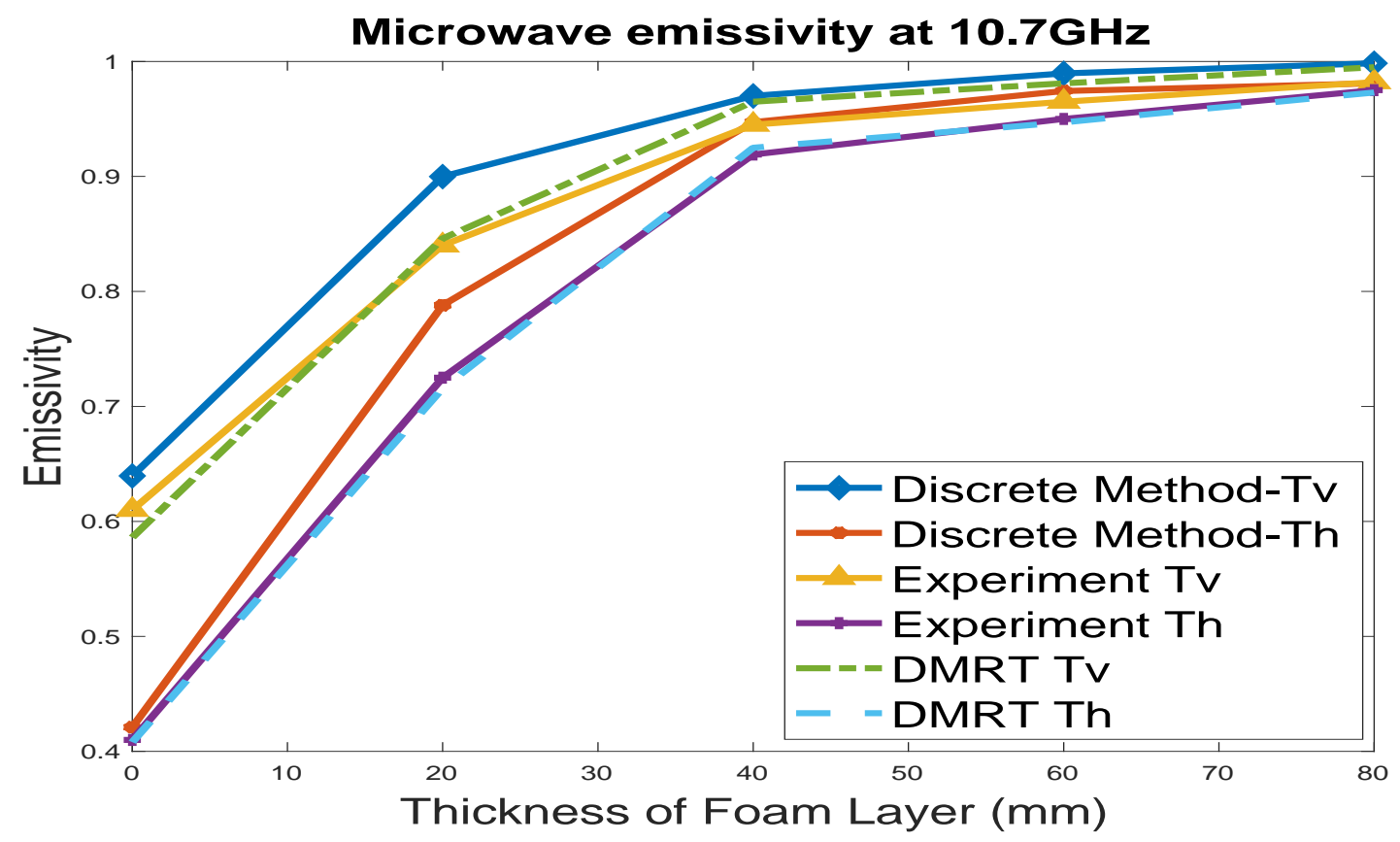

Figure 6. Comparison between simulation results and measurements of microwave emissivity at $10.7 \mathrm{GHz}$ for horizontal and vertical polarization as a function of foam layer thickness.

\section{CONCLUSION}

Absorption is a major contributor to losses in a passive microwave remote sensing as previously reported which suggest that there is negligible scattering due to interaction between EM wave and sea surface covered with foam. This paper illustrates microwave emissions due to electromagnetic wave propagation through layers of sea-foam at WindSat frequencies with accurate prediction of the effective dielectric constant of sea foam based on its microscopic properties. The discrete method used for evaluation of microwave emissions at selected WindSat frequencies follows similar pattern with well-known methods such dense media radiative transfer for both horizontal and vertical polarizations as functions of foam layer thickness and angle of incidence.

\section{ACKNOWLEDGMENT}

This research work was supported financially Dr. Nimibofa Ayawei. 


\section{REFERENCES}

[1] T.T. Wilheit Jr, "A model for the microwave emissivity of the ocean's surface as a function of wind speed," IEEE Trans. Geosci. Electron., vol. GE-17, pp. 244249, April 1979.

[2] P.C. Pandey and R. K. Kakar, "An empirical microwave emissivity model for a foam-covered sea," IEEE J. Oceanic Eng., vol. OE-7, no.3, pp. 135-140, 1982

[3] P. M. Smith, "The emissivity of sea foam at 19 and 37GHz," IEEE Trans. Geosci. Remote Sensing, vol. 26, pp. 541-547, Sept. 1998.

[4] A. Stogryn, "The emissivity of sea foam at microwave frequencies," Journal of Geophysical Research, vol. 77, no. 9, pp. 1658-1666, 1972.

[5] G. F. Williams, "Microwave emissivity measurements of bubbles and foam," IEEE Trans. Geosci. Electron., vol. GE-9, pp. $221-244$, July 1971.

[6] Y. A. Militskii et.al. "Thermal radio emission from foam structures," Sov. Phy. Tech. Phys. Engl. Transl. vol 23, pp. 601-602, 1978.

[7] J. Podzimek, "Size spectra of bubbles in the foam patches and sea salt nuclei over the surf zone," Tellus 36 B(3), pp. 192-202, 1984.

[8] L. A. Rose, W. E. Asher, S. C. Reising, P. W. Gaiser, K. M. St Germain, D. J. Dowgiallo, K. A. Horgan, G. Farquharson, and E. Knapp, "Radiometric measurements of the microwave emissivity of foam," Geoscience and Remote Sensing, IEEE Transactions on, vol. 40, no. 12, pp. 2619-2625, 2002.

[9] A. Camps, M. Vall-Llossera, R. Villarino, N. Reul, B. Chapron, I. Corbella, N. Duffo, F. Torres, J. J. Miranda, R. Sabia, et al., "The emissivity of foam-covered water surface at L-band: Theoretical modelling and experimental results from the frog 2003 field experiment," Geoscience and Remote Sensing, IEEE Transactions on, vol. 43 , no. 5 , pp. 925-937, 2005 .

[10] D. Chen, L. Tsang, L. Zhou, S. C. Reising, W. E. Asher, L. A. Rose, K.-H. Ding, and C.- T. Chen, "Microwave emission and scattering of foam based on MonteCarlo simulations of dense media," Geoscience and Remote Sensing, IEEE Transactions on, vol. 41, no. 4, pp. 782-790, 2003.

[11] X. Z. Huang and Y. Q. Jin, "Scattering and emission from two-scale randomly rough sea surface with foam scatterers," Proc. Inst. Elect. Eng. Microwave Antenna Propagat., vol. 142, pp. 109-114, April 1995.

[12] L. Tsang, C-T. Chen, A. T. C. Chang, J. Guo, and K. H. Ding, "Dense media radiative transfer based on quasi-crystalline approximation with applications to passive microwave remote sensing of snow," Radio Sci., vol. 35, no. 3, pp. 731-749, 2000.

[13] J. Guo, L. Tsang, W. Asher, K.-H. Ding, and C.-T. Chen, "Applications of dense media radiative transfer theory for passive microwave remote sensing of foamcovered ocean," Geoscience and Remote Sensing, IEEE Transactions on, vol. 39, no. 5, pp. 1019-1027, May 2001.

[14] Wei, E. B., and Ge, Y., "A microwave emissivity model of sea surface under wave breaking," Chinese Physics, vol. 34, no. 6, pp. 1259-1264, 2005.

[15] Lui, S. B., Wei, E. B., Hong, J. L., and Ge, Y., "Microwave backscattering from the sea surface with breaking waves," Chinese Physics, vol. 15, no. 9, pp. 2175$2179,2006$.

[16] Wei, E. B., and Liu, Y., "Application of effective medium approximation theory to ocean remote sensing under wave breaking," Science in China Series D: Earth Science, vol. 50, no. 3, pp. 474-480, 2007.

[17] Wei, E. B., "Microwave vector radiative transfer equation of a sea foam layer by the second-order Rayleigh approximation," Radio Science, vol. 46, RS5012, 2011.

[18] Wei, E. B., "Effective medium approximation model of sea foam layer microwave emissivity of a vertical profile," International Journal of Remote Sensing, vol. 34, no. 4, pp. 1180-1193, 2013.

[19] M. D. Anguelova, "Complex dielectric constant of sea foam at microwave frequencies," Journal of Geophysical Research: Oceans (1978-2012), vol. 113, no. C8, 2008.

[20] I. MILITSKII, V. RAIZER, E. Sharkov, and V. Etkin, "Scattering of microwave radiation by foamy structures (honeycomb and emulsion type sea surfs)," Radio Engineering and Electronic Physics, vol. 22, pp. 46-50, 1977.

[21] R. D. Peltzer and O. M. Griffin, "Stability of a three-dimensional foam layer in seawater," Journal of Geophysical Research: Oceans (1978-2012), vol. 93, no. C9, pp. 10804-10812, 1988

[22] Y. Zhang, Y. Yang, and J. Kong, “A composite model for estimation of polarimetric thermal emission from foam covered wind-driven ocean surface," Prog. Electromagn. Res., vol. 37, pp. 143-190, 2002.

[23] M. Y. Koledinstseva, S. K. Patil, R. W. Schwartz, W. Huebner, K. N. Rozanov, J. Shen, and J. Chen, "Prediction of effective permittivity of diphasic dielectrics as a function of frequency," IEEE Transactions on Dielectrics and Electrical Insulation, vol. 16, no. 3, 2009.

[24] S. K. Patil, M. Y. Koledinstseva, R. W. Schwartz, W. Huebner, "Prediction of effective permittivity of diphasic dielectrics using an equivalent capacitance model," Journal of Applied Physics, vol. 104, n0. 7, pp. 074-108, 2008.

[25] Liu, Shu-Bo, En-Bo Wei, and Yan-Xia Jia. "Estimating microwave emissivity of sea foam by Rayleigh method" Journal of Applied Remote Sensing 7.1 (2013): 073598-073598.

[26] Liu, Shu-Bo, et al. "Brightness temperature model of sea foam layer at L-band." Journal of Ocean University of China. JOUC 14.1 (2015): 38.

[27] Chen, C.-T., et al., "Analytical and numerical methods for the scattering by dense media," Geoscience and Remote Sensing Symposium, 2000, Proceedings, IGARSS 2000, IEEE 2000 International, Vol. 5, 2000

[28] Ao, C. O., "Electromagnetic wave scattering by discrete random media with remote sensing applications," Ph.D. Thesis, University of California, Berkeley, Massachusetts Institute of Technology, June. 2001.

[29] M. D. Anguelova, M. H. Bettenhausen, and P. W. Gaiser, "Passive remote sensing of sea foam using physically-based models," in International Geoscience and Remote Sensing Symposium (IGARSS), Denver, CO, USA, pp. 3676-3679, 2006.

[30] J. A. Kong, "Electromagnetic Wave Theory,” Wiley-Inter-science, New York, 704pp.

\section{AUTHORS}

First Author - Ayibapreye Kelvin Benjamin, Ph. D in Computing and Electronic Systems University of Essex, United Kingdom, M.Sc. in Electronic Communications and Computer Engineering, The University of Nottingham, United Kingdom, B.Eng. Electrical/Electronic Engineering, Niger Delta University, Nigeria, Niger Delta University, Wilberforce Island Bayelsa State, Nigeria. ayibapreyebenjamin@ndu.edu.ng

Second Author - Aguiyi Nduka Watson, M.Eng. in Electrical Engineering, Rivers State University, Nigeria, B.Eng. Electrical Engineering, Rivers State University, Nigeria, Federal University Otueke, Bayelsa State, Nigeria. aguiyiwatson@gmail.com

Third Author - Engr. Godday Biowei, Ph. D candidate in the Department of Electrical/Electronic Engineering, Niger Delta University, Wilberforce Island, Bayelsa State, Nigeria. M.Sc. in Electronic Communications and Computer Engineering, The 
University of Nottingham, United Kingdom, B.Eng. Electrical/Electronic Engineering, Niger Delta University, Nigeria, biogod@ndu.edu.ng

Correspondence Author - Ayibapreye Kelvin Benjamin, Ph. D in Computing and Electronic Systems University of Essex, United Kingdom, M.Sc. in Electronic Communications and Computer Engineering, The University of Nottingham, United Kingdom, B.Eng. Electrical/Electronic Engineering, Niger Delta University, Nigeria, Niger Delta University, Wilberforce Island Bayelsa State, Nigeria. ayibapreyebenjamin@ndu.edu.ng, contact number: +2348166924395 